\title{
Early Prediction of Epilepsy Seizures System based on Artificial Immune BCl System
}

\author{
Zaghloul Saad Zaghloul \\ Oil Center Research, LLC \\ LA, USA
}

\author{
Nelly Elsayed \\ The Center of Advanced \\ Computer Science (CACS) \\ Lafayette, LA, USA
}

\author{
Magdy Bayoumi \\ University of Louisiana \\ Lafayette, LA, USA
}

\begin{abstract}
Controlling the surrounding world and predicting future events has always seemed like a dream, but that could become a reality using a Brain Computer/Machine Interface (BCI/BMI). Epilepsy is a group of neurological diseases characterized by epileptic seizures. It affects millions of people worldwide, with $80 \%$ of cases occurring in developing countries. This can result in accidents and sudden, unexpected death. Seizures can happen undetectably in newborns, comatose, or motor impaired patients, especially due to the fact that many medical personnel are not qualified for EEG signal analysis. Therefore, a portable automated detection and monitoring solution is in high demand. Thus, in this study a system of a wireless wearable adaptive for early prediction of epilepsy seizures is proposed, works via minimally invasive wireless technology paired with an external control device (e.g., a doctors' smartphone), with a higher than standard accuracy $(71 \%)$ and prediction time $(14.56 \mathrm{sec})$. This novel architecture has not only opened new opportunities for daily usable BCI implementations, but they can also save a life by helping to prevent a seizure's fatal consequences.
\end{abstract}

\section{General Terms}

Brain Machine Interface, Artificial Immune, Signal Processing.

\section{Keywords}

Brian Computer Interface, BCI, AIS, EEG, Epilepsy Seizure, Detection, Prediction, VLSI.

\section{INTRODUCTION}

Epilepsy is a group of neurological diseases characterized by epileptic seizures that affects more that $10 \%$ of the human population worldwide; nearly $80 \%$ of cases occur in the developing world, and resulted in 116,000 Sudden Unexpected Death in Epilepsy (SUDEP) in the last two years [1]. Epilepsy becomes more common as people age. In developed countries, infants account for most of the seizures. About $5-10 \%$ of people over 80 years old have had a seizure. Sufferers have an increased chance of experiencing a second seizure, and usually epilepsy cannot be cured by [2]. Epilepsy can be a primary cause of sudden death or cause different accidents, especially motor-vehicle accidents [3]. Seizures can also happen to newborns, comatose, or significant motor impaired patients, especially in Intensive Care Units (ICU). The situation can be even more severe when medical personnel are not qualified for EEG signal analysis, which is a very common case. Using online unsupervised BCI, detection and monitoring the solution can at least help in detecting the seizure symptoms early to avoid fatal consequences [4].

Despite the advances in pharmacological treatments, approximately 1 in 3 patients continues to experience frequent seizures. Usually epilepsy cannot be cured; however, medication can control seizures effectively, although in more than $30 \%$ is not effective in generalized seizure and $50 \%$ of people with partial seizures. In the developing world, $75 \%$ of people are not appropriately treated, especially in Africa, where $90 \%$ of the patients do not get treatment at all [5]; the global distribution of seizure cases is shown in Figure 1.

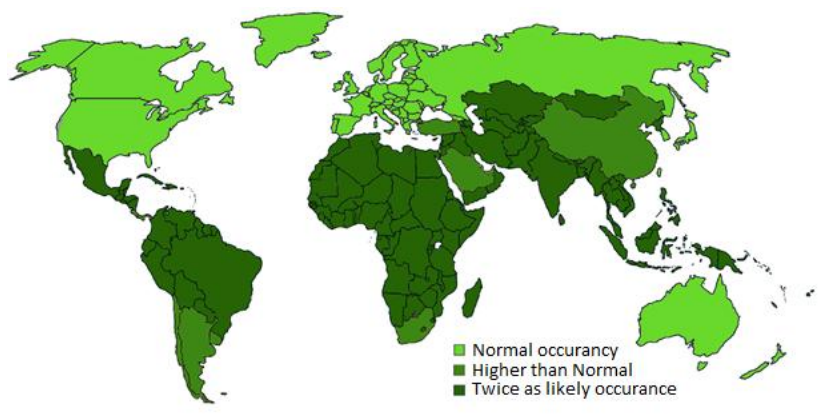

Fig 1: The global epilepsy rate research, University of Oxford in Lancet

The current seizure detection solutions are based on manual inspection, and the demand for automated detection is very high, not to mention the need for prediction. The current stateof-the-art architectures either require a perfect environment to operate, as they are prone to noise, have limitations with predictions due to the static techniques used, or require a powerful computational machines as well as a lot of wiring between the patient and the monitoring station, and in most of the cases, they do not provide a closed loop prediction and detection systems nor easy doctor and ICU personal access to decisions making mechanisms, which in many cases can cost the patient's life.

The study in [6] shows that in most cases the ICU personnel are not qualified for EEG analysis and the majority of patients in the ICU are comatose or have significant motor impairment. The main problem with the current detection systems is that they are only for measuring continuous EEG monitoring devices that are being reviewed by neuroscientists once or twice a day. These devices usually require a lot of wiring, and they do not provide an early prediction or detection feature. Instead they depend solely on the visual inspection of the printed EEG waveform activities. However, the limitations of the detection time are the major challenge. Wireless scalp EEG based early prediction, warning and detection of epilepsy seizure systems were proposed and mentioned by [7] [8]. According to and WHO 2016 [9], a detection system should be:

- Tolerant to noise BCI artifacts and equipment surrounding noise for the ICU room device and the adjacent EMF cross talk and coupling.

- Easy to be used; deployed on different patients from different age groups, and different injury of sickness level, 
as well as connected to warning and alerting device, whether it is a doctor's smart device machine, ICU monitoring device, or a cloud based analysis unit.

- Provides adequate accuracy (more than 60\%) with respect to the agreed seizure detection standard [10].

- Capable of predicting even earlier in the stages of epilepsy seizures, than the current state-of-art $(6.4 \mathrm{sec})$.

- Minimizing the false-positive alarms (VLSI architecture) to be implemented on a chip and a part of the wearable device.

Many proposed seizure detection systems require an "idea signal acquisition," which makes them perform poorly under the presence of additional noises and BCI artifacts [11]. In [12] defined Artificial Immune Systems (AIS) as adaptive problem solving systems, inspired by theoretical biology and immunology functions, Artificial Immune Systems (AIS) are an adaptive clustering and classifier system that is used to detect the abnormality within problem sample space, based on an ideal data set, and the working space of regenerates and mutated samples to compare. These give the AIS a promisingly accurate prediction of behavior, especially for rouge types of input patterns. Therefore, this study proposes an early predictor of epileptic seizures that is based on disposable wearable non-invasive sensors placed on a headband that communicates with a smartphone or any ICU monitoring device via a Bluetooth connection technology.

The proposed system uses a minimally invasive wearable EEG band, with a signal processing chip attached to it, which is placed behind the patient's ear and connects via a Bluetooth technology to an external monitoring device, or to an ICU personnel or doctor's smart handheld device. The results, which include sensitivity and duration parameters configuration are also given to the doctors to set for custom configuration via the prediction smartphone software. It allows the uploading of the raw data and the seizure ictal events recording to the cloud for further research, evidence recording, or tuning the initial population of signature for the detection algorithm. The proposed system is capable of early prediction by an average of $14.56 \mathrm{sec}$, especially for the longlasting seizures (called: status epilepticus by [7]), with accuracy more than $71 \%$ higher than the agreed standards $(>60 \%)$, giving enough time $(>10 \mathrm{sec})$ for warning and treatment [13] [14]. This means that the proposed systems can bring back mobility of a limb (artificial of biological) to a handicapped patient, or can save lives through early prediction of a seizure.

The rest of this work is organized as follows: section 2 will get more familiar with a brief presentation on epileptic seizure; detection and automation technology will also be given. Section 3 is the related work, followed by a brief introduction to the concept fundamentals. In Section 4, the description of the proposed system. Next, the simulation results are given, numerical analysis and discussion. Finally, the authors conclude and discuss future work.

\section{BACKGROUND}

\subsection{Epilepsy seizure types}

According to [15], all the seizures could be divided into two main categories: Partial Seizure and Global Seizure, where it can be subdivided into interlaced subcategories based on the location of the seizure, the patient's visual symptoms, and the awareness state [16].
The authors in [12] proposed regarding seizure detection and for seizure prediction. Additionally, the study in [17] divided seizures into several states:

- Ictal: a physiologic state during a seizure recording. Latin ictus, meaning a blow or a stroke.

- Pre-ictal: the state immediately before the actual seizure or sometimes the beginnings of the ictal state.

- Post-ictal: the state shortly after the seizure.

- Inter-ictal: the period between seizures, or convulsions, that is characteristic of an epilepsy disorder. For most people with epilepsy, it inter-ictal occupies more than $99 \%$ of their life time.

Epileptic seizure is defined as a brief episode of signs or symptoms due to abnormal excessive or synchronous neuronal activity in the brain that can result in uncontrolled movement of the limbs and almost any muscle of the body, and it usually is accompanied by a loss or impair of awareness. It is a disease of the brain characterized by an enduring predisposition to generate epileptic seizures, which are collectively called epilepsy. The study in [18] showed that all epileptic seizures can be classified into six main types:

- Tonic-clonic seizures: characterized by a noticeable contraction of the limbs followed by their extension, along with arching of the back for 10-30 second.

- Tonic seizures: results in a constant contraction of the muscles. The person may even turn blue.

- Clonic seizures: a form of shaking of the limbs; ranges from simple to severe shaking.

- Myoclonic seizures: usually cause spasms of muscles in either a few areas or are generalized through the body.

- Absence seizures: results in only a slight turn of the head or eye blinking where the person often does not fall over, but its post-ictal can last for hours after the seizure ends.

- Atonic seizures: loss of muscle activity for greater than one second; this typically occurs on both body sides.

\subsection{Seizure and EEG}

The authors in [19] defined a seizure as changes in behavior that occur after an episode of abnormal electrical activity in the brain. It could be also defined as convulsions that occur when a person's body shakes rapidly and uncontrollably. During convulsions, the person's muscles contract and relax repeatedly. There are many different types of seizures, even those that have symptoms without shaking. The authors in [20] provided example of different types of seizures and the way that they appear on the EEG recordings, which is basically analyzed via a visual inspection by neuroscientists (all these snapshots of the EEG monitoring of a medical test done at the Department of Neurology, University of Florida and the Atlas for EEG).

\section{RELATED WORK}

\subsection{Statistical Detection}

Unlike in [21], the Amplitude Integrated EEG (aEEG) is widely used to detect neonatal seizures, despite the fact that the accuracy of seizure recognition can be moderate, especially in brief, low amplitude, focally oriented seizures [22]. Neonatologists analyze the aEEG signals at the patient's bedside. In the authors' opinion, clinical neurophysiologists should at least be involved in this interpretation, as they are specially trained in EEG reviewing. Where they compared a four-channel EEG monitoring device with 16 channel EEG 
recordings and found a sensitivity of $68 \%$ and specificity of $98 \%$ with visual interpretation of the signals; the statistical based algorithm is shown in Figure 2.

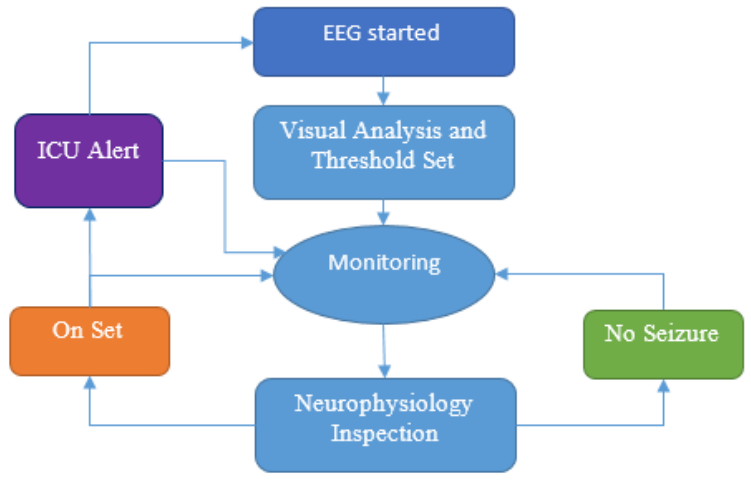

Fig 2: The synchronization likelihood test flow chart

In [23] has experience with another quantitative analysis approach for EEG, namely the synchronization likelihood (SL), where a nonlinear measure of statistical interdependencies between time series, which has shown to be a promising measure for detecting seizures in neonatal EEGs and frontal lobe epilepsy. They used a SL based technique to design an online automatic detection method for real-life EEG seizure monitoring. The experiment used the EEG data from 50 patients over a 15 -month period, measuring how five were suffering from seizures. Using the 16 channel EEG, the architecture was able to detect most of the seizure with $68 \%$ sensitivity and specificity of $98 \%$.

Despite of the accurate results and faster performance, this architecture suffers from several drawbacks that limit its implementation as a wearable device. The first disadvantage is that it requires a near ideal operation scenario as this design is prone to noise and BCI artifacts. The second disadvantage, which is mentioned by the authors, is that the frame structure scanned could produce a high rate of false positives especially in the case of a scalp EEG input electrode.

\subsection{SVM Seizure Detection Techniques}

There are several works that focus on Epileptic Seizure detection. The authors in [15] addresses the computational and implementation challenges associated with detecting seizure onset with an implantable device. The study specifically shows how a Two-Class Support Vector Machine (SVM) can be used to synthesize patient-specific detectors that outperform a patient non-specific detector. The study also discusses other methods that enable efficient implementation of the discriminator functions produced by the SVM algorithm.

The researchers claim that: "the detector extracts, from each channel, features that can be used to infer the presence of seizure activity. Since spectral energy has been shown to be useful in the context of intracranial seizure detection, the chosen features where the energy within the frequency bands $0-16 \mathrm{~Hz}$ and $15-37 \mathrm{~Hz}$ ". Regardless of the low power achievements this design has several drawbacks, including that the usage of only static patient related thresholds for seizure detection, requires an invasive implants, which are very sensitive to noise and almost fits the lower bound of the acceptable false alarms.

\subsection{Pattern Recognition Based Detection}

The authors in [23] present a novel event-based seizure detection algorithm along with a low-power digital circuit.
Using an invasive depth-electrode, recordings from rats are used to validate the algorithm and hardware performance. The main detection algorithm is shown in Figure 3.



Fig 3: Flow chart of the operation stage of the algorithm

The research also discusses the main problems and cost in translating mathematical models into hardware implementations. It also claims they could reach an average TPR of $95.3 \%$ and FPR of $88.9 \%$. They implemented their system on a CMOS circuit drawing $\sim 350 \mathrm{nW}$ of power source with $250 \mathrm{mV}$.

\subsection{Adaptive Prediction Algorithm}

The experiments in [8] discuss from a signal processing point of view, that a data analysis and prediction scheme can be considered to be a four-step process of signal enhancement, including adaptive autoregressive modeling and prediction, envelope detection and a binomial decision rule. Their proposed architecture is based on scalp EEG electrode for data acquisition. A block diagram of the real-time adaptive seizure prediction algorithm that is based on Wiener algorithm is implemented in real-time Figure 4.



Fig 4: Block diagram of the real-time prediction

After the amplification stage, the band-pass infinite impulseresponse filter $(10-500 \mathrm{~Hz})$ attenuates the low frequency noise and the higher frequency artifacts that could lead to false positive detection of seizures [5]

As a summery, most of the previous works are based on a computational intense algorithm or not suitable for VLSI implementation due to the area and power limitations [17], and most of them are proposed for seizure detection but do not focused on the early prediction of the pre-ictal stage. Additionally, they do not solve threshold update problem, the artifact noise attenuation, the detection bounded minima problem, the static pattern storage, not the framing bounders problem, respectively. 


\section{PROPOSED SYSTEM}

\subsection{Proposed Method Concepts}

The proposed system uses a non-invasive wearable EEG band, with a signal processing chip attached to it, which connects via Bluetooth technology to an external monitoring device, ICU personnel or doctor's smart handheld device. The sensitivity and duration parameters configuration are also given to the doctors to set for custom configuration via the smartphone software.

\subsubsection{Cauchy-based filter}

Many proposed seizure detection systems require ideal signal acquisition, which makes them perform poorly under the presence of additional noises and BCI artifacts. The study in [24] shows that more than $90 \%$ of the affecting noises of the scalp EEG recording comes from the EOG and EMG artifacts, where the rest comes from non-acceptable electrode conductivity, loose electrode connections, the surrounding electrical resonance, adjacent electric and magnetic fields EMF and wire crosstalk.

Wang et al. [24] studied different noise reduction and elimination techniques to avoid the Gaussian assumption of the stationary noise, and found the best results of false detection rate (FDR) are $0.15 / \mathrm{h}$ and $0.08 / \mathrm{h}$ for the overall and best case of the experimental scenario, where they had life EEG data from ten human patients. That was achieved via using a Cauchy-based observation together with the autoregressive (AR) model to represent the state transitions. Thus as soon as noise and artifacts invade the EEG signal, especially closer to the tail, the probability value of the detection will be close to zero, as a property of the Gaussian distribution. This is because the Cauchy distribution (1) has a higher probability density function (PDF) than the Gaussian case.

$$
f\left(x ; x_{0}, \gamma\right)=\frac{1}{\pi \gamma\left[1+\left(\frac{x-x_{0}}{\gamma}\right)^{2}\right]}
$$

Where $x_{0}$ is the location parameter, it is specifying the location of the peak of the distribution, and $\gamma$ is the scale parameter (i.e. called the probable error). Obviously, the maximum value or amplitude of the Cauchy PDF is $1 / \pi \gamma$ which is located at the peak. Thus, relying on the Cauchy distribution instead of the Gaussian helps to clearly distinguish between misleading the merged signals. Either it is a seizure event or a system of most known artifacts. The authors also aware that this filtering could be compromised in the case of overlapping several simultaneous artifacts and/or seizure occurrences; however, from literature these scenarios happen are very rarely in the usual practice.

\subsubsection{Artificial Immune System}

The study in [25] discussed the history of AIS and showed that at late 1970's there was a considerable interest in biology as a source of inspiration for solving computational problems. Models of the central nervous system have driven artificial neural networks. The Darwin Theory spawned evolutionary simulations in natural selection. According to Dasgupta [26], the definition of the biological immune system (IS) is "an accurate and elaborate defense system that consists of multilayers of protection where each layer provides different types of defense mechanisms for find, detect, recognize and response for foreign organisms and pathogens".

\subsubsection{Negative Selection Mechanism}

In [27] the Negative selection is defended as a process of selection that takes place in the thymus gland. Its main purpose is to provide tolerance for self-cells. It deals with the immune system's capability to detect unknown antigens while not reacting to self-cells. The maturation of the T-cells is very simple. T-cells are exposed to self-proteins in a binding process. If this binding activates the T-cell, then the T-cell is killed, otherwise it is allowed into the lymphatic system [28].

\subsubsection{AIS Definition}

Artificial Immune System (AIS) is concerned with computational methods inspired by the process and mechanisms of the biological immune system. The scope of artificial immune systems could be thought to be restricted to pattern recognition tasks. The basic negative selection algorithm approach proposed by [29] as shown in Figure 5.

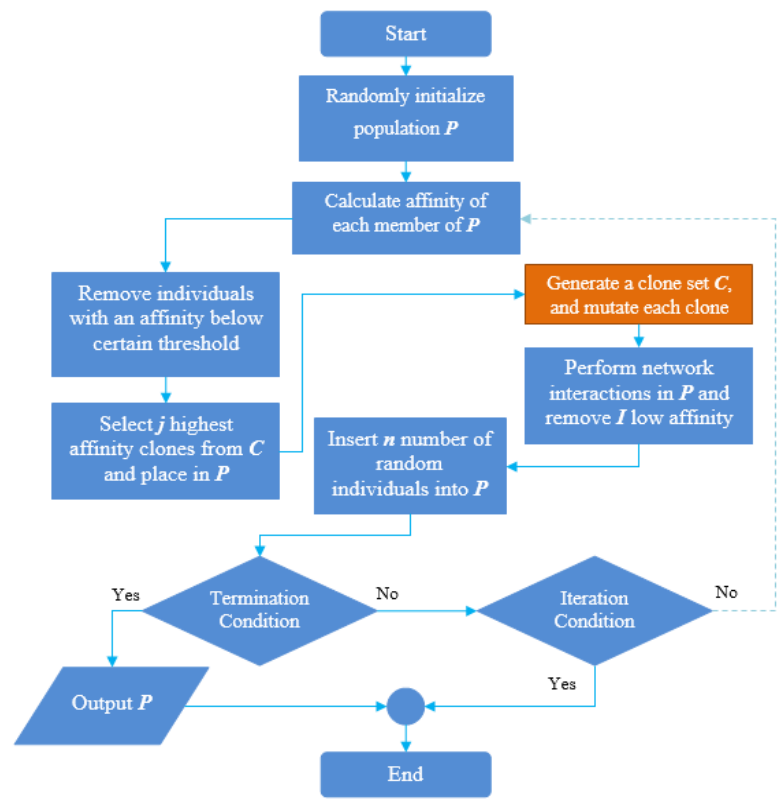

Fig 5: Flow chart of the real-time AIS algorithm

\subsection{Proposed Architecture}

The system solution diagram is shown in Figure 6. The gray arrow path represents the input data manipulation path, the blue arrow represents the control lines, the orange arrows represent the parameter configuration lines, and the green dotted line represents the wireless line to the outside control device.

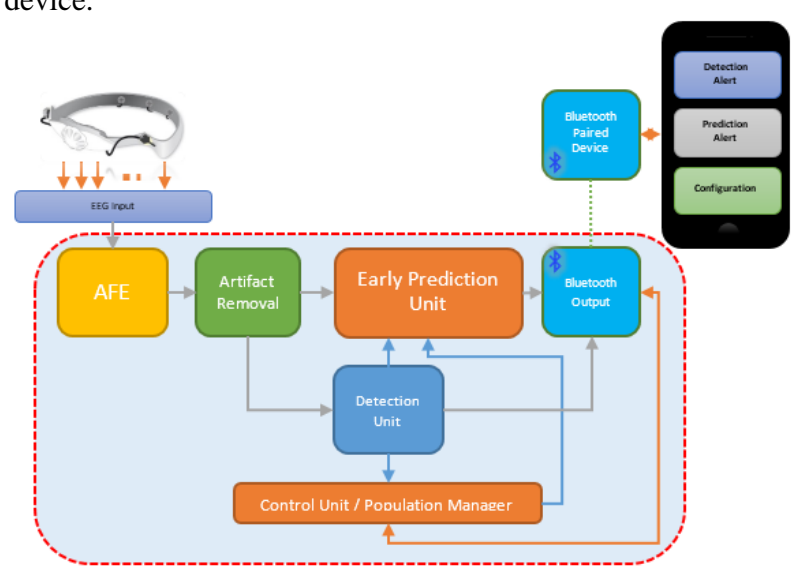

Fig 6: The proposed solution logical block diagram 
The proposed architecture can logically be divided into three main stages, the Signal Conditioning, the Adaptive Signal Analysis, and the Decision Making stages. The Signal Condition consists of four units: The Analog Front-End (AFE), the Digital Wavelet Transform (DWT), the Signature Generation Unit (SGU), and the Artifact Removal Unit (ARU). Those are followed by the Adaptive Signal Analysis that is consistent of the Seizure Detection Unit (SDU) and the Seizure Prediction Unit (SPU). Finally, it will be followed by the Decision Making stage that consists of the Decision Controller and the RF Module, which is the gateway between the system on the chip and the control interface on the doctor's smart handheld device.

\subsubsection{Signal Conditioning Stage}

This stage is the first part of the online system where the input is the raw EEG from four input channels which is amplified 100 times, then processed via a band pass filter through a 30$100 \mathrm{~Hz}$ band to cutoff the high frequency. It is sampled over $250 / 500 \mathrm{~Hz}$ inside the AFE unit with resolution of 16 bit analog to digital converter (ADC) then it is fed to the Cauchy based ARU.

This paper used the unit described in [24] as the main artifacts removal block in the adaptive early prediction unit. Then, the signal is sent to the DWT unit to smooth the signal and reduce the sample space dimensions. After W cycles (where W represents the width of signature window) the record width is selected empirically to optimize the chip area and the signature detection of the signal's amplitude envelope, it is set to 8 Signature Units (Sig.) after the analysis of the simulation results and an adequate value for the window length is shown, such as in Figure 7. Thus, regarding the dominant spiking channel, a behavior signature is generated in the local channel data and the affected adjacent channel simultaneously as described in the previous work [30].

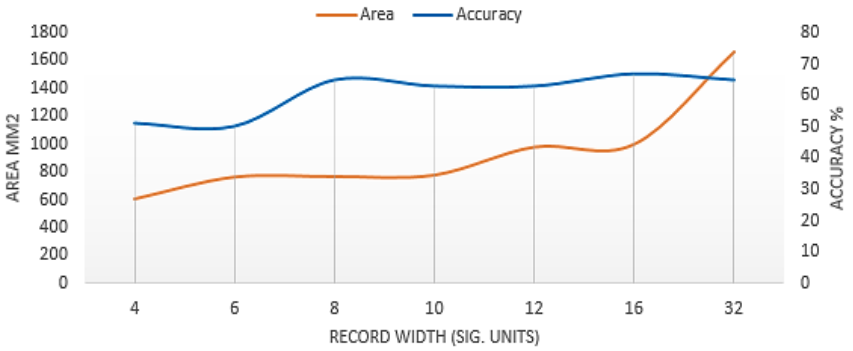

Fig 7: The empirical simulations between record width $W$, accuracy and estimate area

\subsubsection{Adaptive Signal Analysis Stage}

In this stage the signatures and the input signals are parallel fed in into two main units: the Seizure Detection and the Seizure Early Prediction units. The seizure detection unit uses an enhanced version (tolerant of artifact noise) of the technique described in [23]. The system detects the diversity for the standard deviation with combining the resulted value matrix of size $N \times N$, where $N$ is the number of channels, then calculates the max of the signal likelihood values. Then the likelihood value is compared to the adaptive threshold and duration (number of clock cycles of occurrence) to detect a seizure. The Detection Unit can also be done via the AIS algorithm which could enhance the detection accuracy by $17 \%$; however, this will cause an increase in the chip area by $57 \%$ and its role as a backup plan strategy will not be used with respect to the Early Predicting Unit. The Prediction Unit principal engine of the parameterized AIS algorithm is shown in Figure 8.
The main detection engine algorithm is based on the negative selection algorithm idea where it tries to find the abnormality in the system for every rogue input (antigen). The starting point of this algorithm is to produce a set of self-waves, $S$, that define the normal state of the system. The task then is to generate a set of detectors, $D$, that only bind/recognize the complement of $S$.

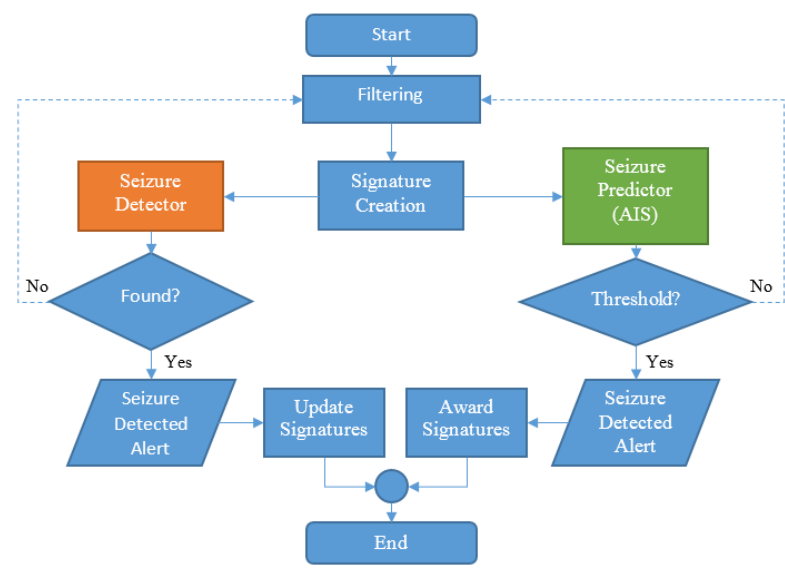

Fig 8: The proposed analysis algorithm flowchart

The algorithm's clonal selection theory has been used as inspiration for the development of AIS that perform computational optimization and pattern recognition tasks. In particular, inspiration has been taken from the antigen driven affinity maturation process of B-cells, with their associated mutation mechanism. These AIS also often use the idea of memory cells to retain good solutions to the problem being solved. The pseudo code for the clonal selection is shown in Figure 9.



Fig 9: The pseudo code for the Clonal selection algorithm

The affinity $d$ measurement is determined using the Euclidean distance of the antigen with the detectors is defined by:

$$
d(S, D)=\sum_{i=1}^{n}\left(S_{i}-D_{i}\right)^{2}
$$

Using the adaptive behavior of the architecture with the mutation shuffling dramatically reduces the local minima scenario and increases the true negative detection (e.g. specificity) from the usual pattern recognition scenario by more than $12 \%$.

\subsubsection{Decision Making Stage}

This is responsible for sending the warning signal of the predicted seizure. It is wanted for existing seizures or transferring the EEG data to the external monitoring device or warning appliance. It compares the output of the seizure detection unit and the seizure prediction unit with respect to the time and the current sliding window, and it choose to either warn for the upcoming possible pre-ictal, or alarm for the existing seizure-ictal. Also, this unit reads the threshold, which is the signal sensitivity parameter from that external 
device. Starting with the EEG input registers and the AFE units. Then, as soon as a seizure prediction hits the threshold, a warning signal is generated and sent to the output unit stating the time and the window of the EEG that caused the firing to the external device. On the other hand, if a seizure onset is detected, an alarm is sent to the external device and an update to the population manager is sent to either add the previous signature if it does not exist, or increase the priority weight of the existing signature.

\subsection{Simulation Results}

Initially the algorithm was experiencing a training phase. This simulation used Matlab, Simulink, EEGLAB and WaveClus for the initial simulation of the algorithm and decided on the initial values of the threshold window width and the parameters wire data width to select the width and the constant parameters of the thresholds to compare them to the FPR. After 5 min of simulation and by studying the different threshold values of the accuracy of the system, then the initial value was selected of the combined likelihood value between 0.20 and 0.25 with a mean of 0.23 . This is slightly higher than the previous work by $33.3 \%$ due to deployment of the AR unit; the value was selected to optimize the FPR, as shown in Figure 10, where that the relatively stable lower FPR and the highest TPR in the approximate range can be found, as described in [13] [23], where the initial threshold was selected to be.

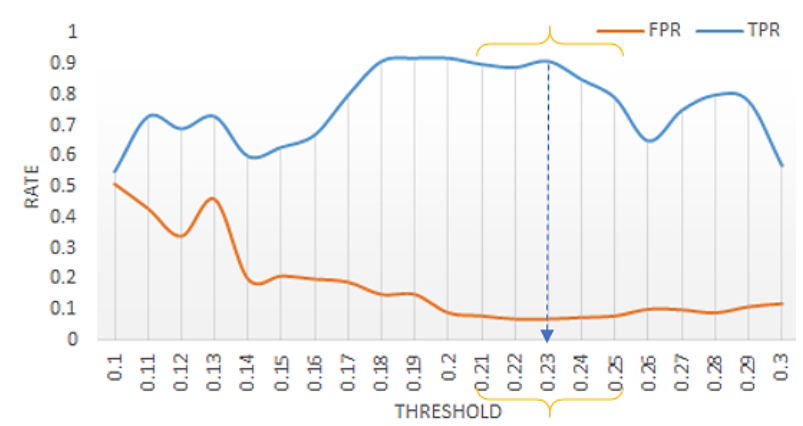

Fig 10: The correlation between FPR and TPR

We are aware it is not the optimal mathematical solution for the minimization of the FPR and the maximization of the TPR to be optimized, but this approximate solution provides a decent initial value for the threshold, where the rest is up to the doctor for fine tuning of the system regarding each individual patient or special situation. The next step was to generate the initial population of signatures of the AIS. The data were used from CHB-MIT lab [31], where an experiment was done on 24 patients at a Boston Hospital in December 2010 for an average of two hours. Then a randomly selected 8 patients' data to act as training data. Next, the rest of the data were used for the testing and verification phase containing seizure and seizure free recordings. The data consist of scalp EEG recordings of pediatric group subjects with intractable seizures. Subjects were monitored for up to several days following the withdrawal of anti-seizure medication in order to characterize their seizures and assess their candidacy for surgical intervention; the patient's statistics are shown Figure 11, and are according to the expert's visual inspection of the EEG recordings. These parameters were used to determine the initial value for the configuration parameters that define the number of the initial antibody set, the number of the generated clones of each antibody, the gene count (i.e., matching samples), the mutation cycle length, the value of the threshold to the antigen and the signature selection, shown in Table 1.
These parameters were used to determine the initial value for the configuration parameters that define the number of the initial antibody set, the number of the generated clones of each antibody, the gene count (i.e., matching samples), the mutation cycle length, the value of the threshold to the antigen and the signature selection, shown in Table 1.

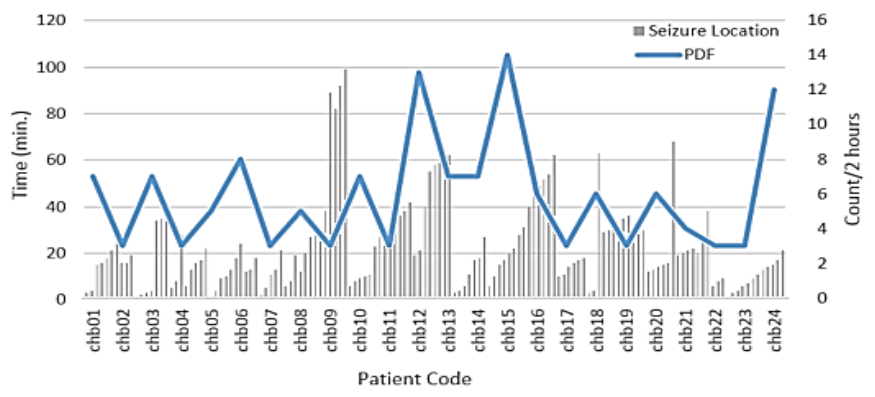

Fig 11: Selected patients characteristics

These parameters were used to determine the initial value for the configuration parameters that define the number of the initial antibody set, the number of the generated clones of each antibody, the gene count (i.e., matching samples), the mutation cycle length, the value of the threshold to the antigen and the signature selection, shown in Table 1.

Table 1. AIS initial learning parameters

\begin{tabular}{l|l}
\hline Parameter & Value \\
\hline Number of antibody's & 50 \\
Number of clones & 25 \\
Genes & 255 \\
Mutation Cycles & 83 \\
Remove Threshold & 0.3 \\
Selection Threshold & 0.01 \\
Diversity & 0.64 \\
\hline
\end{tabular}

The learning curve of the initial likelihood threshold of the detection algorithm with respect to the FPR and the TPR was set using two hundred input segments, each of $5 \mathrm{sec}$ duration that included a seizure onset event for not less than $3 \mathrm{sec}$ each.

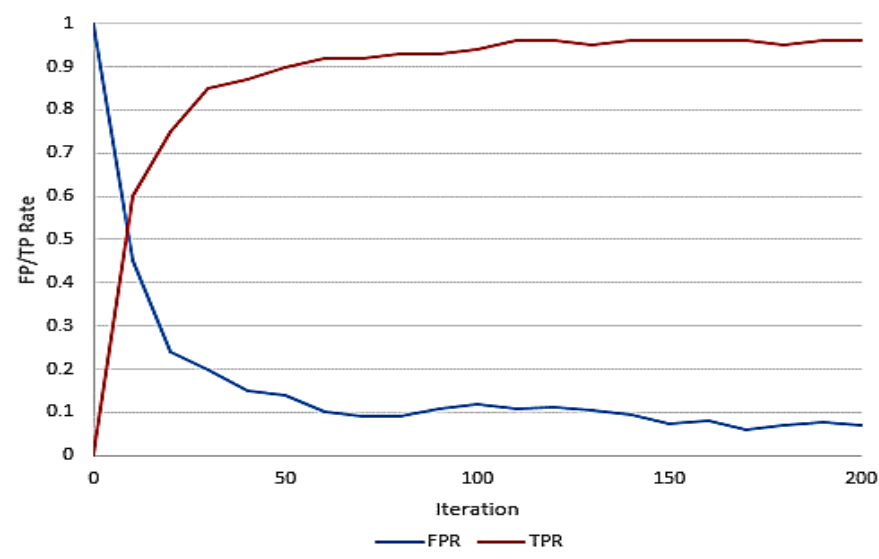

(a) 


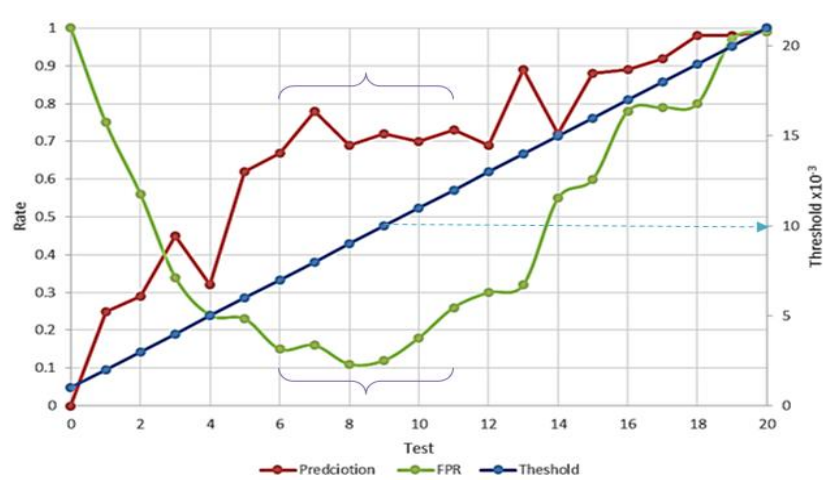

(b)

Fig 12: (a) The learning curve of initial (td) of FPR and TPR; (b) The initial prediction threshold

This was done to ensure a variable location of the ictal event within the sample space for the detection mechanism input. The results are shown in Figure 12 (a).

For the prediction unit, the threshold $(t p)$ of the detector's likelihood of prediction was set empirically with a twenty test segment, which was taken from the previous data sample for $20 \mathrm{sec}$ right before the seizure ictal event. The event was marked via the visual inspection of the neuroscientist (given with the data). The initial threshold was set via the imperial test, which was similar to the method used to determine the initial value of $t d$ in regards to the correlation between the FPR and the TPR, as shown in Figure 12 (b).

The values of the td and $t p$ are initially set to 0.23 , and 0.09 respectively. These values were chosen via the imperial test and serves as a guideline for the system to function initially. However, the endpoint management application will give the ability for the doctors of the ICU to tune these parameters to the sensitivity of the warning and the alarm thresholds which suits their need for every different case, situation or patients' needs. After the training phase was completed, several patients' EEG data were used, excluding the previously selected eight patients who were used for training. The simulation ran for a period of $5 \mathrm{~min}$ each, where the prediction time threshold was initially set from $10-15 \mathrm{sec}$, and the system was able to predict and detect seizures for most of the selected patients with a sensitivity of $71 \%$ and an accuracy of $91 \%$. The results of the experiments are shown in Table 2.

Table 2. Selected patients for testing phase characteristics

\begin{tabular}{lcccccccc}
\hline Patient Code & PT1 & PT2 & PT3 & PT4 & PT5 & PT6 & PT7 & PT8 \\
\hline Genre & Male & Female & Female & Male & Female & Female & Female & Female \\
Age (years) & 22 & 17 & 10 & 7 & 3.5 & 12 & 9 & 6 \\
E.S./2 hours & 3 & 5 & 3 & 6 & 5 & 3 & 3 & 12 \\
Predicted & 5 & 6 & 3 & 8 & 9 & 4 & 4 & 14 \\
Detected & 4 & 5 & 3 & 6 & 10 & 4 & 3 & 11 \\
T-to-S (sec) & 12.4 & 16.32 & 16.6 & 10.8 & 13.4 & 18.3 & 17.2 & 11.6 \\
\hline
\end{tabular}

After the simulation step, a verification procedure took place. And comparing the proposed early prediction algorithm to a simple random guess from a normal distribution. The test that was proposed and used to verify the prediction step was performed on logical decisions bases, not a random guess. After that the proposed System II was compared to the previously proposed architecture Arch1, and Arch2, proposed in [8] and [23], respectively.

\subsection{Results Analysis}

First, all of the previous work and the proposed work exceed the performance of the random guess which makes them all a selective candidate for future implementation, although, Arch. 1 and Arch. 2 provided an acceptable average result (following the prediction standards stated by [14]). It was noticed that Arch.1 has the lowest average performance compared to Arch. 2 and the proposed method. After analysing the situation, it turns out that Arch.1 would have performed better if the authors would have proposed a filter as it is in the case in the other two systems, which makes the results biased. However, the comparison took place to the proposed system with the stated systems exactly as is. On the other hand, the authors in [32] claimed to describe that a dog may warn of impending psychogenic non-epileptic seizures (PNES), saying that such a dog's companionship may cut down on seizure frequency. Also they mentioned that the ability for a dog to obtain help during or after a seizure could prove lifesaving. Further, they showed in their study that a patient was "alerted by his dog 7 minutes prior to having psychogenic seizures." However, the experiment was carried with a success rate of four out of six parliaments. Additionally, a strong criticism by [33] for that study. Thus, a brief, comprehensive summary of the state-of-the-art systems, existing claimed methods, and the nerve stimulation implant are listed, in addition to the proposed system on Table 3.

Table 3. Comprehensive prediction summary

\begin{tabular}{|l|l|l|l|l|l|}
\hline Comparison & Claim & SNA & Arch l & Arch 2 & Proposed \\
\hline Detection & N/A & Yes & Yes & Yes & Yes \\
\hline Prediction & Yes & No & No & Yes & Yes \\
\hline Input & N/A & Implant & Implant & Scalp EEG & Scalp EEG \\
\hline Average time & 7 min & -- & -- & $6.45 e c$ & 14.57 sec \\
\hline Detection Accuracy & N/A & $65 \%$ & $65 \%$ & $91 \%$ & $92 \%$ \\
\hline Prediction Accuracy & $66.7 \%$ & -- & -- & $63 \%$ & $72 \%$ \\
\hline Hardware & -- & Implant & Yes & Yes & Yes \\
\hline Learning & N/A & N/A & $\begin{array}{l}\text { Threshold } \\
\text { Adaption }\end{array}$ & $\begin{array}{l}\text { Static SVM } \\
\text { Seizure patterns }\end{array}$ & $\begin{array}{l}\text { Adaptive AIS } \\
\text { with adaption }\end{array}$ \\
\hline Filtering & N/A & N/A & No & $\begin{array}{l}\text { Frequency based } \\
\text { for High Freq. } \\
\text { Artifacts }\end{array}$ & $\begin{array}{l}\text { Cauchy Based } \\
\text { (EOC, EMG) }\end{array}$ \\
\hline
\end{tabular}

As a summary, the authors have shown, illustrated and verified that this novel proposed system is capable of early prediction of several types of epileptic seizure, with a prediction period average of $14.57 \mathrm{sec}$, twice that of the stateof-the-art devices [34], with an average accuracy of $72 \%$ and a lower FPR than the current system by $75 \%$. However, there are several disadvantages or undeveloped situations which the proposed system at this stage cannot handle. The first issue is the abnormal artifacts that usually exit with several infants who suffer from seizure. Infant Spasm Syndrome, and Febrile Seizure, which usually occur in children aged 3 months to 5 years, are examples. They usually occur with a high fever. However, such cases are considered to be rare (i.e. $2 \%$ to $5 \%$ of all the children). The second issue is that there is a relatively short prediction time compared to the claimed dogs' ability to predict seizures (if this clam could be proven). However, the authors believe that the advances in neurosciences can bring us more clues and more input data signal markers that can be extracted to predict a better pattern of information for a more accurate prediction within the EEG data. 


\section{CONCLUSIONS AND FUTURE WORKS}

We propose an early prediction and detection system for epileptic seizures. Regarding the problem statement for the epilepsy detection issue the proposed system was able to solve almost all of the pending issues with the detection technology. It proposed a novel - not only a seizure detection system - but early epilepsy prediction feature that uses a parameterized AIS based adaptive prediction system that was verified and outstood the state-of-the-art architectures through prediction accuracy by $114 \%$, the false positive rate by $(75 \%)$ and the average prediction time by more than twice the current prediction time.

The main detection problem and issue are the surgical procedures, the noise proof design, and the high false alarm rate issues, were solved via the proposed wearable and disposable wireless band with minimally invasive dry EEG sensors for signal acquisition; the system is EOC and EMG artifacts-proof due to the support of the Cauchy based artifact filter; the accurate parameterized AIS prediction mechanize, respectively. Making the proposed system not only a promising early prediction system architecture but a novel BCI based solution for many brain disorders' early prediction and detection systems.

For future work, the promising results of the medical markers of the stress affected person's EEG recording gives a new opportunity for designing a Stress Detection Warning Application that could be used for office and business fields to detect stressed personnel early enough before the loss of use and performance. Thus, a BCI approach for early stress detection, early reduced alert/sleep detection and early Alzheimer diagnosis would be the next steps in this work for a better tomorrow for the whole of humanity.

\section{ACKNOWLEDGMENTS}

Our special thanks to Filip, Ali and John Rahat for their help with the Matlab simulation and implementation; great appreciation for Jaqueline Hebert for the review.

\section{REFERENCES}

[1] Kiran, G., et al., 2014. Design, implementation and evaluation of a brain-computer interface controlled mechanical arm for rehabilitation. I2MTC, 1326-1328.

[2] Hitiris, N., Mohanraj, R., Norrie, J., Brodie, M., 2007. Mortality in Epilepsy. J. of Epilepsy Behavior (March 2007), 363-376.

[3] Marx, J., et al., 2013. Rosen's Emergency Medicine. Concepts and Clinical Practice, 8th ed. Elsevier, ch.18.

[4] Eadie, M., 2012. Shortcomings in the current treatment of epilepsy. Expert Review of Neurotherapeutics (December 2012), 1419-1427.

[5] Longo, L., 2012. 369 Seizures and Epilepsy. Harrison's principles of internal medicine, 18th ed., McGraw-Hill.

[6] Labiner, D., et al., 2010. Essential services, personnel, and facilities in specialized epilepsy center. Epilepsia PubMed J. (November 2010), 2322-2333.

[7] Rogowski, Z., Gath, I., Bental, E., 1981. On the prediction of epileptic seizures. Biology Cybernetic (January 1981), 9-15.
[8] Rajdev, P., Ward, M., Rickus, J., Worth, R., Irazoqui, P., 2010. Real-time seizure prediction from local field potential using an adaptive Wiener algorithm. Computer in Biology and Medicine, 97-108, Elsevier.

[9] World Health Organization, 2016, Epilepsy Fact sheet. WHO, Retrieved 4 (March 2016).

[10] William, C., Litt, B., 2008. Technology Insight: Neuroengineering and Epilepsy_-Designing Devices for Seizure Control. N. Clinical Practice Neuro., 190-201.

[11] Nicolas-Alonso, L., Gomez-Gil, J., 2012. Brain Computer Interfaces, a Review. J. Sensors, 1211-1279.

[12] Castro, L., Timmis, J., 2002. Artificial Immune Systems: A New Computational Intelligence Approach, 57-58.

[13] Haas, S., Frei, M., Osorio, I. 2007. Strategies for adapting automated seizure detection algorithms. Med. Eng. and Physics, vol. 29, no. 8, 895-909.

[14] Kristin, K., Theoden, I., et al., 2011. Early Seizure Detection. J. of Clinical Neurophysiology (March 2013), pp. 259-268, ACNS.

[15] Simon, D., Greenberg, A., Michael, J., Roger, P., 2012. Clinical Neurology. 8th Edition, McGraw-Hill Medical.

[16] Fisher, S., Acevedo, C., Arzimanoglou, A., et al., 2014. ILAE official report: A practical clinical definition of epilepsy. J. of Epilepsia (April 2014), 475-482.

[17] Cecotti, H., Graser, A., 2011. Convolutional Neural Networks for P300 Detection with Application to BCI. IEEE T. on Pattern Analysis and Machine Intelligence, (March 2011).

[18] Quyen M, et al., 2001. Anticipation of epileptic seizure from Standar EEG Recordings. J. of Lance.

[19] Krumholz, A., et al., 2007. Practice parameter: evaluating an apparent unprovoked first seizure in adults: Report of the Quality Std. Neurology, 9, 1991-2007.

[20] Ludres, HO., Noachtar, S., 2000. Atlas and Classification of Electroencephalograph.1st ed., Elsevier.

[21] Maynard, D., Prior, PF., Scott DF., 1969. Device for continuous EEG monitoring of cerebral activity in resuscitated patients. Br. Med Journal, 545-546.

[22] Young, GB., et al., 2009. Seizure detection with a commercially available bedbased EEG monitoring and subhailine montage. Neuocrit Care 2009.

[23] Ponten, S., et al., 2010. Feasibility of online seizure detection with continuous EEG monitoring in the intensive care unit. Seizure, Science Direct, 580-586.

[24] Wang, Y., et al., 2015. A Cauchy-Based State-Space Model for Seizure Detection in EEG Monitoring System. IEEE Intelligent System J., Brain Machine Interface.

[25] Hassan, Y., Younes, A., Elsayed, N., 2013. Artificial immune system and soft computing. Int. J. of IJCSITS, 250-258.

[26] Dasgupta, D., 1999. An overview of artificial immune systems and their applications, in Dasgupta. Springer Berlin Heidelberg, 3-21. 
[27] Timmis, J., 2007. Artificial Immune Systems - Today and Tomorrow. Natural Comp. (January 2007), 1-18.

[28] Nguyen, VT., Kha, NV., Anh, N., 2012. Research Some Algorithms in Machine Learning and Artificial Immune System. J. of IJCSI, (March 2012), 477-251.

[29] Esponda, F., Forrest, S., Helman, P., 2004. A formal framework for positive and negative detection schemes. IEEE T. on Systems, Man and Cybernetics, 7, 346-363.

[30] Zaghloul, ZS., Bayoumi, M., 2015. Adaptive neural matching online Spike Sorting VLSI chip design for wireless implants. ICASSP 2015.

[31] AL Goldberger F., et al., 2000. Physio Bank, Physio Toolkit, and Physio Net: Components of a New Research
Resource for Complex Physiologic Signals. Circulation, vol. 101 , no. $23,215-220$.

[32] Vito, DI., et al., 2010. A seizure response dog: video recording of reacting behaviour during repetitive prolonged seizures. Epileptic Disorder, 142-145.

[33] Doherty, M., Haltiner, A., 2007. Wag the dog: Skepticism on seizure alert canines. J. of Neurology (October 2007), 262.

[34] Avestruz, A., et al., 2008. A 5uW/Channel Spectral Analysis IC for Chronic Bidirectional Brain-Machine Interfaces. J. of Solid-State Circuits, (September 2014), 3006-23024. 